\title{
VISCOUS-FLOW CALCULATIONS FOR KVLCC2 IN DEEP AND SHALLOW WATER
}

\author{
SERGE TOXOPEUS* \\ *Maritime Research Institute Netherlands (MARIN) / Delft University of Technology \\ P.O. Box 28 \\ 6700 AA Wageningen, The Netherlands \\ e-mail: s.l.toxopeus@marin.nl, web page: http://www.marin.nl
}

Key words: KVLCC2, manoeuvring, shallow water, CFD, RANS

\begin{abstract}
In the SIMMAN 2008 workshop, the capability of CFD tools to predict the flow around manoeuvring ships has been investigated. It was decided to continue this effort but to extend the work to the flow around ships in shallow water. In this paper, CFD calculations for the KLVCC2 are presented. The aim of the study is to verify and validate the prediction of the influence of the water depth on the flow field and the forces and moments on the ship for a full-block hull form.

An extensive numerical investigation has been conducted. For each water depth, several grid densities were used to investigate the discretisation error in the results. In general, the uncertainties were found to increase with increased flow complexity, i.e. for larger drift angles or yaw rates. A dependency of the uncertainty on the water depth was not found. The predicted resistance values were used to derive water-depth dependent form factors. Comparisons with resistance measurements and with an empirical formula given by Millward show good agreement for deep as well as for shallow water depths. The CFD results give insight into the forces and moments acting on the ship as a function of the drift angle, yaw rate and water depth. A clear dependence of the forces and moments on the water depth is found for steady drift conditions. For pure rotation, this dependence is much more complex and only develops fully for larger non-dimensional rotation rates. The paper shows that CFD is a useful tool when studying the flow around ships in restricted water depths.
\end{abstract}

\section{INTRODUCTION}

In the SIMMAN 2008 workshop [1], the capability of CFD tools to predict the flow around manoeuvring ships has been investigated. Within the NATO RTO Applied Vehicle Technology group on Assessment of Stability and Control Prediction Methods for NATO Air \& Sea Vehicles (AVT-161) it was decided to continue this effort but to extend the work to the flow around ships in shallow water, see e.g. Toxopeus [2]. Accordingly, the KVLCC2 hull form was selected, since for this ship captive model tests in various water depths are available. In this paper, CFD calculations performed by MARIN for the KLVCC2 are presented. The aim of the study is to investigate the uncertainty in predicting the forces and moments on a full-block hull form in various water depths; validate the predictions using model test results; and to obtain information about the influence of the water depth on the flow around the ship and the forces and moments on the hull. 


\section{COORDINATE SYSTEM}

The origin of the right-handed system of axes used in this study is located at the intersection of the water plane, midship and centre-plane, with $x$ directed forward, $y$ to starboard and $z$ vertically downward. The forces and moments presented in this paper are given according to this coordinate system.

In the present calculations, a positive drift angle $\beta$ corresponds to the flow coming from port side (i.e. $\beta=\arctan -v / u$ ). The non-dimensional yaw rate $\gamma$ is calculated with $\gamma=r \cdot L_{\mathrm{pp}} / V$ and is positive for a turn to starboard when sailing at positive forward speed.

\section{$3 \quad$ KVLCC2}

The KVLCC2 (KRISO Very Large Crude Carrier) hull form was one of the subjects of study during the CFD Workshops Gothenburg 2000 [3] and 2010 [4] and the SIMMAN 2008 Workshop [1]. For straight ahead conditions, the flow features and resistance values were measured, see Lee et al. [5] and Kim et al. [6].

Captive model tests for the bare hull KVLCC2 were conducted by INSEAN in preparation for the SIMMAN 2008 Workshop [1], see also Fabbri et al. [7, 8] and Campana and Fabbri [9]. A set of PMM tests comprising amongst others the measurement of the forces and moments for steady drift motion and oscillatory yaw motion was performed. During the tests, the model was free to heave and pitch. For the present work, only the tests with the bare hull form are considered.

\section{VISCOUS FLOW SOLVER AND COMPUTATIONAL SETUP}

\subsection{ReFRESCO}

REFRESCO is a MARIN spin-off of FRESCO [10], which was developed within the VIRTUE EU Project together with Technische Universität Hamburg-Harburg (TUHH) and Hamburgische Schiffbau-Versuchanstalt (HSVA). REFRESCO is an acronym for Reliable and Fast Rans Equations solver for Ships, Cavitation and Offshore. It solves the multi-phase unsteady incompressible RANS equations, complemented with turbulence models and volume-fraction transport equations for each phase. The equations are discretised using a finite-volume approach with cell-centred collocated variables. The implementation is face-based, which permits grids with elements with an arbitrary number of faces (hexahedrals, tetrahedrals, prisms, pyramids, etc.). The code is targeted, optimised and highly validated for hydrodynamic applications, in particular for obtaining current, wind and manoeuvring coefficients of ships, submersibles and semi-submersibles $[11,12,13,14]$. For all cases presented in this study the $y^{+}$values in the first cell from the wall are below 1, such that the equations are integrated down to the wall.

\subsection{Turbulence closure models}

Several different turbulence closure models are available in REFRESCO. In this study, the SST version [15] of the two-equation $k-\omega$ turbulence model is used. In the turbulence model, the Spalart correction (proposed by Dacles-Mariani et al. [16]) of the stream-wise vorticity can be activated. 


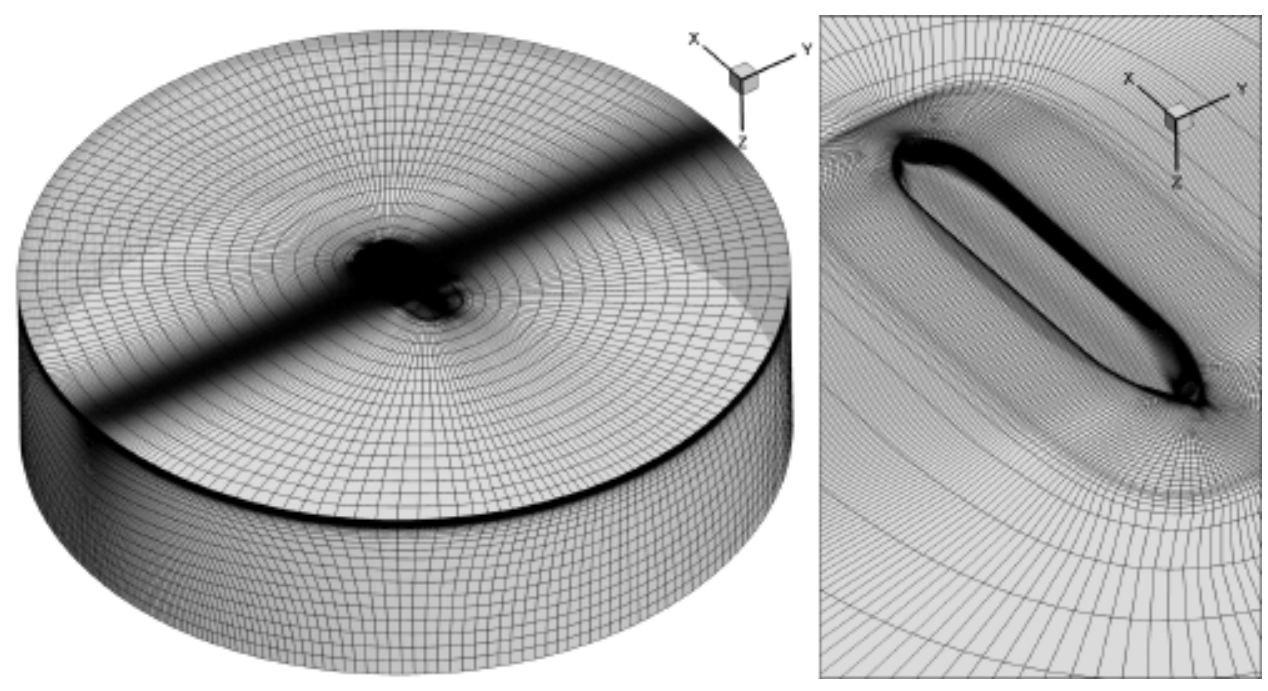

Figure 1: Example grid, KVLCC2, deep water (coarsened for presentation)

\subsection{Implementation of rotational motion}

For ship manoeuvres, not only oblique flow is of interest, but also the flow around the ship when it performs a rotational (yaw) motion. In RANS, the rotational motion can be modelled in several ways, such as moving the grid in a rotational motion through a stationary flow (inertial reference system), or by letting the flow rotate around the stationary ship (non-inertial reference system). For this work a non-inertial reference system is chosen. Centrifugal and Coriolis forces to account for the rotation of the coordinate system are added to the momentum equation as source terms. More information about the implementation can be found in Toxopeus [14].

\subsection{Grid generation}

For best performance of REFRESCO, multi-block structured O-O grids are used for this study. Grid points have been clustered towards the hull surface and bottom to ensure proper capturing of the boundary layers. The far field boundary is generated as a cylindrical surface, to facilitate the use of a single grid for all computations. An example grid is given in Figure 1.

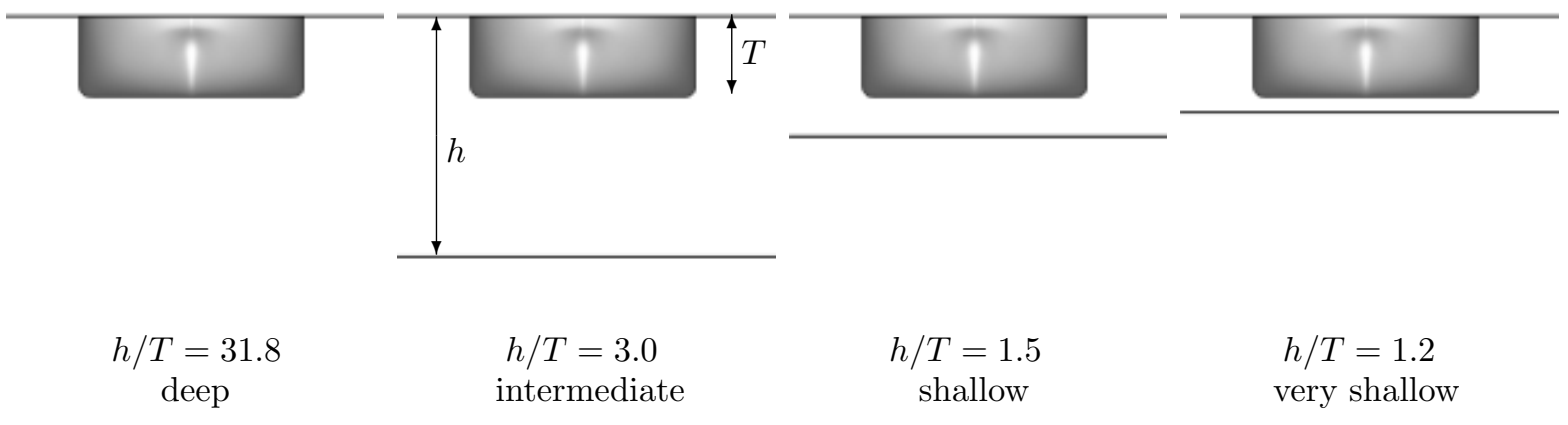

Figure 2: Water depth to draught ratios considered in this study 
Grids were generated with GridPro for four different water depth $h$ to draught $T$ ratios, i.e. $h / T=31.8$ representing deep water, $h / T=3.0$ representing an intermediate water depth, $h / T=1.5$ representing shallow water and $h / T=1.2$ representing very shallow water, see Figure 2. Basically, the grid topology around the hull for the four water depths was the same, the only difference being the addition of grid blocks between the bottom of the hull and the sea floor for each water depth.

Based on these grids, geometrically similar grids were generated using GridPro in order to be able to assess the discretisation errors and to accelerate the iterative procedures by using coarse grid solutions as initial flow fields for fine grid computations. Additional grids are obtained by coarsening the finest grid in all directions. Table 1 lists the grid densities used for this study.

Table 1: Overview of grid densities

\begin{tabular}{|l|l|}
\hline$h / T$ & Grid cells $\left(\times 10^{-3}\right)$ \\
\hline 31.8 (Deep) & $12721,8455,5388,3340,2270,1590,121$ \\
3.0 (Intermediate) & $13005,8597,5573,3446,2374,1604,137$ \\
1.5 (Shallow) & $11659,7688,4936,3106,2112,1437,119$ \\
1.2 (Very shallow) & $11031,7270,4664,2899,1999,1351$ \\
\hline
\end{tabular}

\subsection{Boundary conditions}

The calculations presented in this study were all conducted without incorporating free-surface deformation. Based on the speeds used during the tests and the range of drift angles or rotational rates studied, the effects of Froude number and free-surface deformation on the forces on the manoeuvring ship are likely to be reasonably small and assumed to be smaller than the uncertainties due to e.g. discretisation errors or errors in the experimental results. To simplify the calculations, symmetry boundary conditions were therefore applied on the undisturbed water surface and dynamic sinkage and trim was neglected. On the hull surface, no-slip and impermeability boundary conditions are used. For all calculations, even for deep water, the boundary condition on the bottom surface is set to moving-wall/fixed $\operatorname{sip}\left(\bar{u}=\bar{V}_{\infty}\right)$.

Calculations for ships at drift angles or rotation rates are conducted by setting the boundary conditions at the exterior to the proper inflow velocities. This is done using the BCAutoDetect boundary condition, which automatically applies inflow conditions or outflow (Neumann) conditions on the cell faces, depending on the normal velocity at each cell face on the boundary. Therefore, the computational domain does not need to be changed for each new calculation and a single grid for different manoeuvring conditions can be used. Details about BCAutoDetect can be found in Toxopeus [14].

\subsection{Acceleration of the calculations}

In order to efficiently generate results for many drift angles, a routine was used to automatically increment the drift angle during a single simulation. Simulations begin with a pre-set drift angle, until a specified number of iterations is reached, or when the maximum change in the residuals is less than a specified convergence criterion. Next, the 
drift angle is incremented by $\Delta \beta$, by changing the inflow conditions, and the solution is continued from the solution from the previous drift angle. Starting the calculations from a converged solution at a slightly different drift angle saves time compared to performing each calculation separately from undisturbed flow. This procedure is repeated until the desired maximum inflow angle is reached. In Toxopeus [14], it is demonstrated that this approach provides the same results as those obtained with multiple single-drift angle calculations.

This procedure was designated drift sweep and the application has already been presented in e.g. Toxopeus [14], Vaz et al. [12] and Bettle et al. [17].

\section{PROGRAMME OF CALCULATIONS}

Most calculations were conducted for a Reynolds number of $R e=3.7 \times 10^{6}$ which corresponds to the Reynolds number during the INSEAN model tests (see $[7,8,9]$ ). The conditions are specified in Table 2. Sinkage and trim and free surface deformation were not taken into account. Furthermore, it was assumed that the flow domain was not restricted in horizontal direction, i.e. the basin walls were neglected. During the measurements, the model was free to sink and trim and basin walls were present. Especially for the shallow water conditions, this may lead to differences between the model test results and the computations.

Table 2: Overview of computations

\begin{tabular}{|l|c|c|c|c|}
\hline Condition & Deep & Intermediate & Shallow & Very shallow \\
& $h / T=31.8$ & $h / T=3.0$ & $h=1.5$ & $h / T=1.2$ \\
\hline Drift & $5388 \times 10^{3}$ Cells, & $5573 \times 10^{3}$ Cells, & $4936 \times 10^{3}$ Cells, & $4464 \times 10^{3}$ Cells, \\
sweep & $0^{\circ}-32^{\circ}, 2^{\circ}$ incr. & $0^{\circ}-32^{\circ}, 2^{\circ}$ incr. & $0^{\circ}-32^{\circ}, 2^{\circ}$ incr. & $0^{\circ}-38^{\circ}, 2^{\circ}$ incr. \\
\hline$\beta=0^{\circ}$ & all 7 grids & all 7 grids & all 7 grids & all 6 grids \\
\hline$\beta=4^{\circ}$ & all 7 grids & all 7 grids & all 7 grids & all 6 grids \\
\hline$\gamma \neq 0$ & $12721 \times 10^{3}$ Cells, & all 7 grids & all 7 grids & all 6 grids \\
& $0.1-0.6,0.1$ incr., 0.65 & $0.1-0.6,0.1$ incr. & $0.1-0.6,0.1$ incr. & $0.1-0.6,0.1$ incr. \\
\hline$\gamma=0.4$ & all 7 grids & all 7 grids & all 7 grids & all 6 grids \\
\hline
\end{tabular}

Additional calculations were conducted for the deep water condition, at straight ahead sailing and a Reynolds number of $R e=4.6 \times 10^{6}$. This condition was chosen in order to be able to compare the REFRESCO results with wind-tunnel measurements of the flow field around the KVLCC2, see Lee et al. [5] and with towing tank experiments performed by Kim et al. [6]. The calculations comprised the change of Reynolds number and variations in the turbulence modelling.

\section{PRESENTATION AND DISCUSSION OF THE RESULTS}

\subsection{Iterative error}

All calculations were run until the maximum normalised residual res $\max _{\text {ax }}$ (the so-called $L_{\infty}$ norm) between successive iterations had dropped well below $1 \times 10^{-5}$ or when further iterative convergence was not obtained. The changes in the non-dimensional integral quantities (forces and moments) were well below $1 \times 10^{-7}$. A representative convergence 
history of the residuals and the changes in the forces for a calculation on the finest grid is presented in Figure 3.
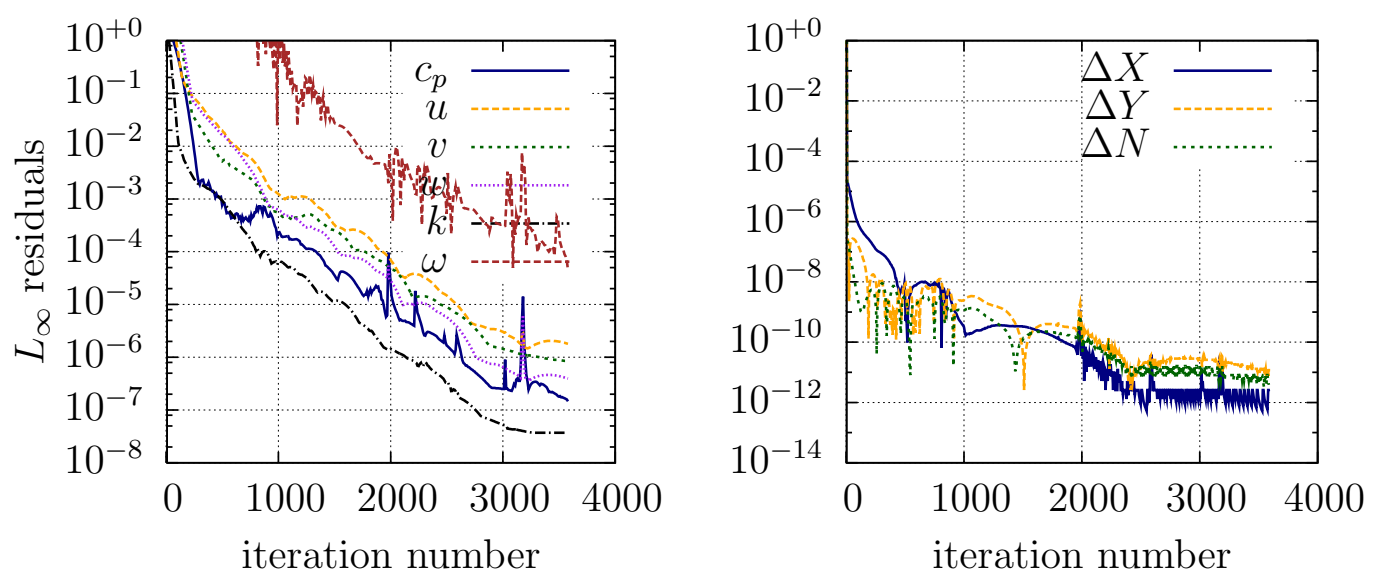

Figure 3: Iterative convergence, deep, $\beta=4^{\circ}$

\subsection{Discretisation error}

Using the procedure proposed by Eça et al. [18], the uncertainties in the forces and moments are estimated. Based on an analysis of the results for each grid, it was decided to use the 5 finest grids for the uncertainty analysis. The number of grids $n_{g}$ used depended on the scatter in the results for the coarsest grids. It was found that for grids with a relative step size $h_{i}$ of 2 and above, the results are not consistent with the finer grid results. This means that with the present grid layout, grids of more than about $1.6 \times 10^{6}$ cells are required to obtain a reliable solution of the forces and moments. As an example of the verification results, plots are presented for $\beta=4^{\circ}$ with deep and very shallow water in Figure 4.

Verification studies have been performed for all other calculations with $\beta=0^{\circ}, \beta=4^{\circ}$ and $\gamma=0.4$, but the results have not been included in this paper. These studies have indicated that the uncertainties for the rotational motion cases are higher than for the pure drift cases $\left(\beta=0^{\circ}\right.$ and $\left.\beta=4^{\circ}\right)$. This can be attributed to the increased complexity of the flow. Especially for the large rotation rates $(\gamma \geq 0.4)$, the uncertainties increase. For rotational motion, the uncertainties in $X$ and $Y$ are large, while the uncertainty in $N$ is reasonable. This is probably caused by the fact that during pure yaw motion, the yaw moment (sum of contributions) is better defined than the longitudinal force or side force (difference between contributions). The theoretical order of convergence should be 2 for REFRESCO. However, due to flux limiters, discretisation of the boundary conditions and other factors, the apparent order of convergence is expected to be between 1 and 2 for geometrically similar grids in the asymptotic range. Considering uncertainty estimates for the various water depths and conditions, the apparent orders do not always follow this expectation. This indicates that either even finer grids are required, or that scatter in the results spoils the uncertainty estimate. A relation between the uncertainties and the water depths was not found. 

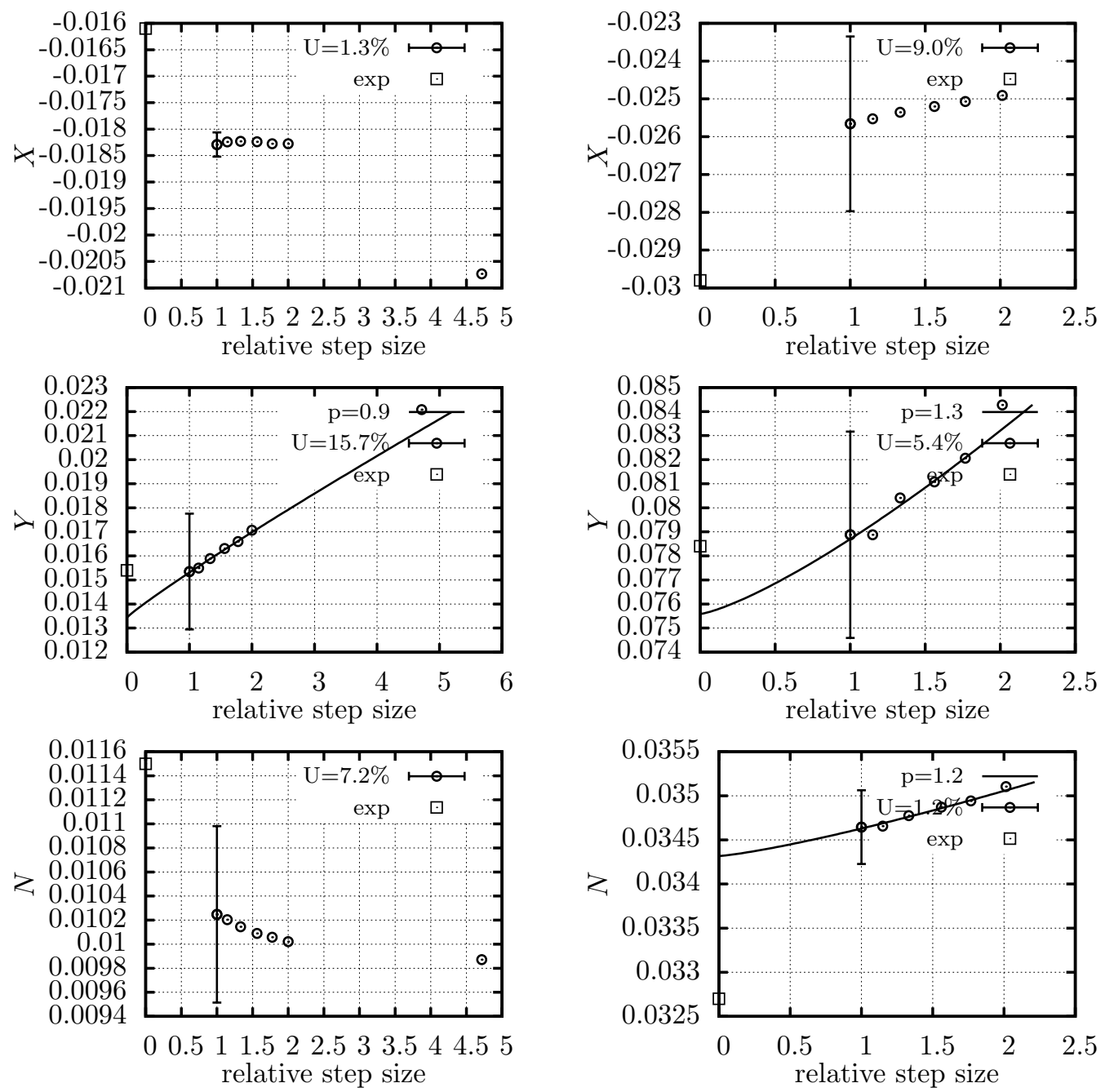

Figure 4: Uncertainty analysis, deep (left) and very shallow (right) water, $\beta=4^{\circ}$

Additionally, the influence of the grid density on the flow field at the propeller plane has been investigated. It was seen that with increasing grid density, the agreement between the CFD results and the experiments becomes qualitatively slightly better, but the hook shape in the propeller plane as visible in the experiments [5] is not well resolved (see Figure 6). The sensitivity of the flow field to grid refinement is judged to be small. Between densities of $3340 \times 10^{3}$ and $12721 \times 10^{3}$, the flow field does not change significantly. Looking at the differences between the solutions obtained on the six grids, it is not thought that the solution will improve upon grid refinement and therefore modelling errors are expected to exist in the CFD results. Alternative turbulence models might improve the results, as was shown for e.g. PARnAssos during the Gothenburg 2010 CFD Workshop [19]. 

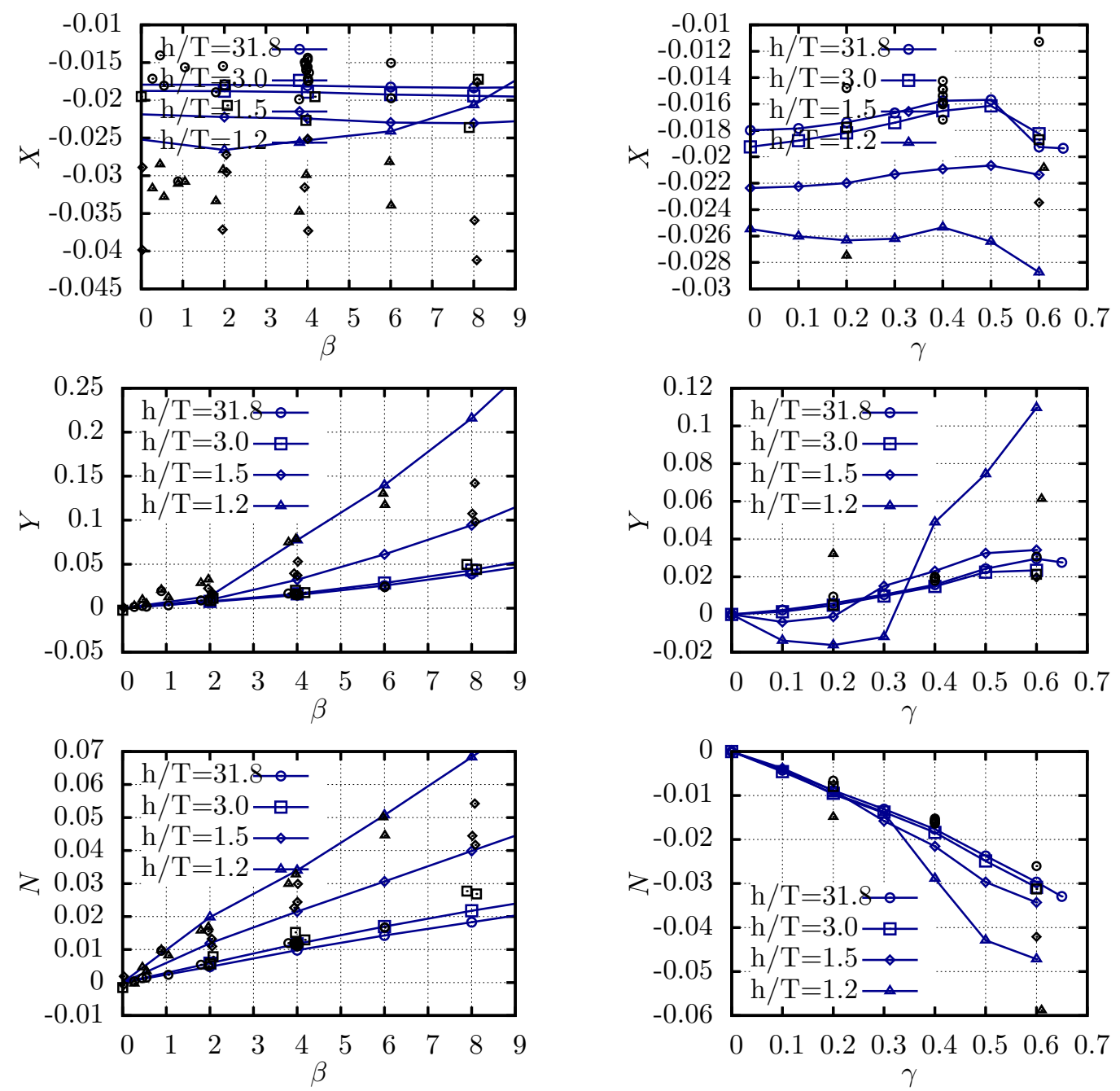

Figure 5: Comparison between calculations (blue lines with symbols) and experiments (black symbols), relation with $\beta$ (left) and with $\gamma$ (right)

\subsection{Comparison with the experiments}

\subsubsection{Manoeuvring conditions}

Comparisons between the CFD results and the experiments are shown in Figure 5. In general, the agreement is qualitatively reasonable, but quantitatively, considerable differences are seen. In most cases, validation of the solution is not achieved, which indicates that modelling errors are present in the simulations or that the uncertainties claimed for the experimental results are optimistic. When the solution is validated, the level of validation is generally large, e.g. larger than $10 \% D$. Especially for the $X$ force, discrepancies are found and the trends do not appear to be represented well. Similar results and deviations from the trends were obtained by Zou [20]. Reasons for the discrepancies might be the neglect of free surface and dynamic trim and sinkage. However, large scatter exists in the experimental data and therefore the uncertainty in the experiments is expected to be relatively large. Furthermore, it is questioned whether the false bottom used during the 
tests was sufficiently sealed at all sides to correctly model shallow water conditions and whether blockage of the basin walls influences the results, see also Simonsen et al. [21]. This should be investigated further.

\subsubsection{Straight ahead sailing}

For the calculation for deep water, $\beta=0^{\circ}$ and $R e=4.6 \times 10^{6}$, comparisons were made with the experiments performed by Lee et al. [5] and Kim et al. [6]. The agreement between the CFD results and the experiments is good for the two most upstream planes $\left(x=-0.35 L_{\mathrm{pp}}\right.$ and $x=-0.4 L_{\mathrm{pp}}$, not presented here). Further downstream, differences appear which increase when going aft. The comparison for $x=-0.4825 L_{\mathrm{pp}}$ is shown in the top half of Figure 6. As discussed in section 6.2, it was expected that improvements can be made by selecting alternative turbulence modelling. This assumption was tested using additional calculations in which the Spalart correction of the streamwise vorticity according to Dacles-Mariani et al. [16] was activated. With this correction, the strain rate $S$ is replaced by $S_{c}=\Omega+C \cdot \min (0, S-\Omega)$ in which the constant $C$ should be chosen, see Eça [22]. In the present calculation with active correction, $C$ was set to 10 (which is far outside the normal range of application), and the results are given in the lower half of Figure 6. It is seen that with this correction, the agreement between the computations and the experiments improves considerably, which demonstrates the sensitivity of the results to the turbulence modelling. These findings correspond to those of Eça [22] and Eça et al. [19], based on results obtained with the viscous-flow solver PARNASSOS.

Table 3: Resistance prediction, deep water, $\beta=0^{\circ}, \gamma=0$

\begin{tabular}{|l|c|r|r|r|r|}
\hline & $\begin{array}{c}n_{\text {cells }} \times 10^{-3} \\
\text { (Half ship) }\end{array}$ & $\begin{array}{r}C_{T} \\
\times 10^{3}\end{array}$ & $\begin{array}{r}C_{F} \\
\times 10^{3}\end{array}$ & $\begin{array}{c}C_{P V} \\
\times 10^{3}\end{array}$ & $\mathrm{E}(\% D)$ \\
\hline Experiments [6] & - & 4.110 & - & - & - \\
Experiments (KVLCC2M) [23] & - & 4.152 & - & - & 1.0 \\
Mean G2010 [4] & - & 4.180 & - & - & 1.7 \\
PARNASSOS [19] & 6000 & 4.077 & 3.325 & 0.752 & -0.8 \\
REFRESCO & 6361 & 4.185 & 3.457 & 0.728 & 1.8 \\
REFRESCO (DM C=10) & 6361 & 4.109 & 3.280 & 0.829 & 0.0 \\
\hline
\end{tabular}

In Table 3, the predicted resistance in deep water is compared to previous calculations and to the experiments ${ }^{1}$ (indicated by $D$, with $U_{D}=1.0 \% D$ ) performed by MOERI [6]. Additionally, results from tests by Kume et al. [23] with the KVLCC2M hull form (identical to the KVLCC2 except for some fairing of the propeller shaft) are added $\left(U_{D^{\prime}}=3.3 \% D^{\prime}\right)$. The KVLCC2M resistance value has been corrected for the difference in Reynolds number, using a form factor $(1+k)=1.2$. In order to compare with other resistance predictions for the KVLCC2 found in literature, the values in the

\footnotetext{
${ }^{1}$ During the Gothenburg 2010 CFD workshop, it was concluded that the MOERI experiments were performed with the rudder attached to the model. Therefore, the G2010 KVLCC2 case contributions comprised calculations including the rudder, while the present calculations are for the bare hull. The KVLCC2M test results were obtained for the bare hull.
} 

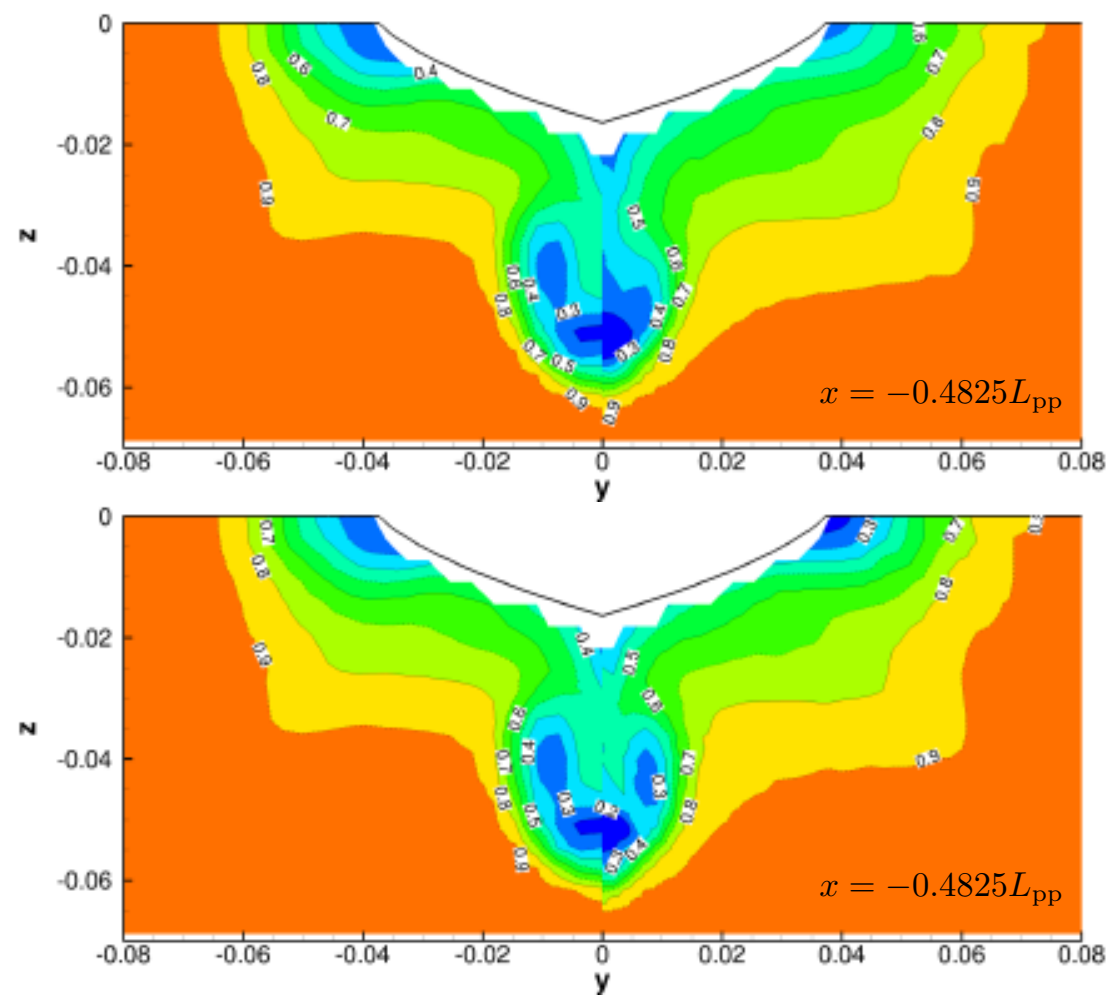

Figure 6: Comparison of flow field between experiments (left) and calculations (right), without (top) and with (bottom) correction for vorticity, deep water, $\beta=0^{\circ}$

table have been made non-dimensional using the wetted surface. The total resistance is indicated with $C_{T}$, the friction component by $C_{F}$ and the pressure component by $C_{P V}$.

The resistance predicted by REFRESCO is about $1.8 \%$ higher than the value found in the experiments. Assuming that the numerical uncertainty $U_{S N}$ is the same for $R e=$ $4.6 \times 10^{6}$ as for $R e=3.7 \times 10^{6}$, i.e. $U_{S N}=U_{I}+U_{G} \approx U_{G}=1.3 \% S$, the validation uncertainty is about $U_{V}=\sqrt{U_{D}^{2}+U_{S N}^{2}} \approx 1.6 \% D$. The validation uncertainty and the comparison error for the uncorrected REFRESCO results are found to be of similar orders of magnitudes, although strictly validation of results is not obtained.

The Spalart streamwise vorticity correction used to improve the wake field also leads to a reduction of the comparison error. However, although the streamwise vorticity correction appears to improve the results, care should be taken with this modification since it is not guaranteed that the correction will lead to better results for other conditions as well. It just demonstrates that the turbulence modelling can have a large impact on the computational results.

\subsection{Influence of water depth}

\subsubsection{Forces and moments}

In Figure 7 and Figure 8 the forces and moments as a function of the drift angle and rotation rate are shown for each water depth. The influence of the water depth is already evident in these graphs at $h / T=3.0$. For the forces and moments as a function of the drift angle $\beta$, consistent trends are found. The relation between the forces and moments 

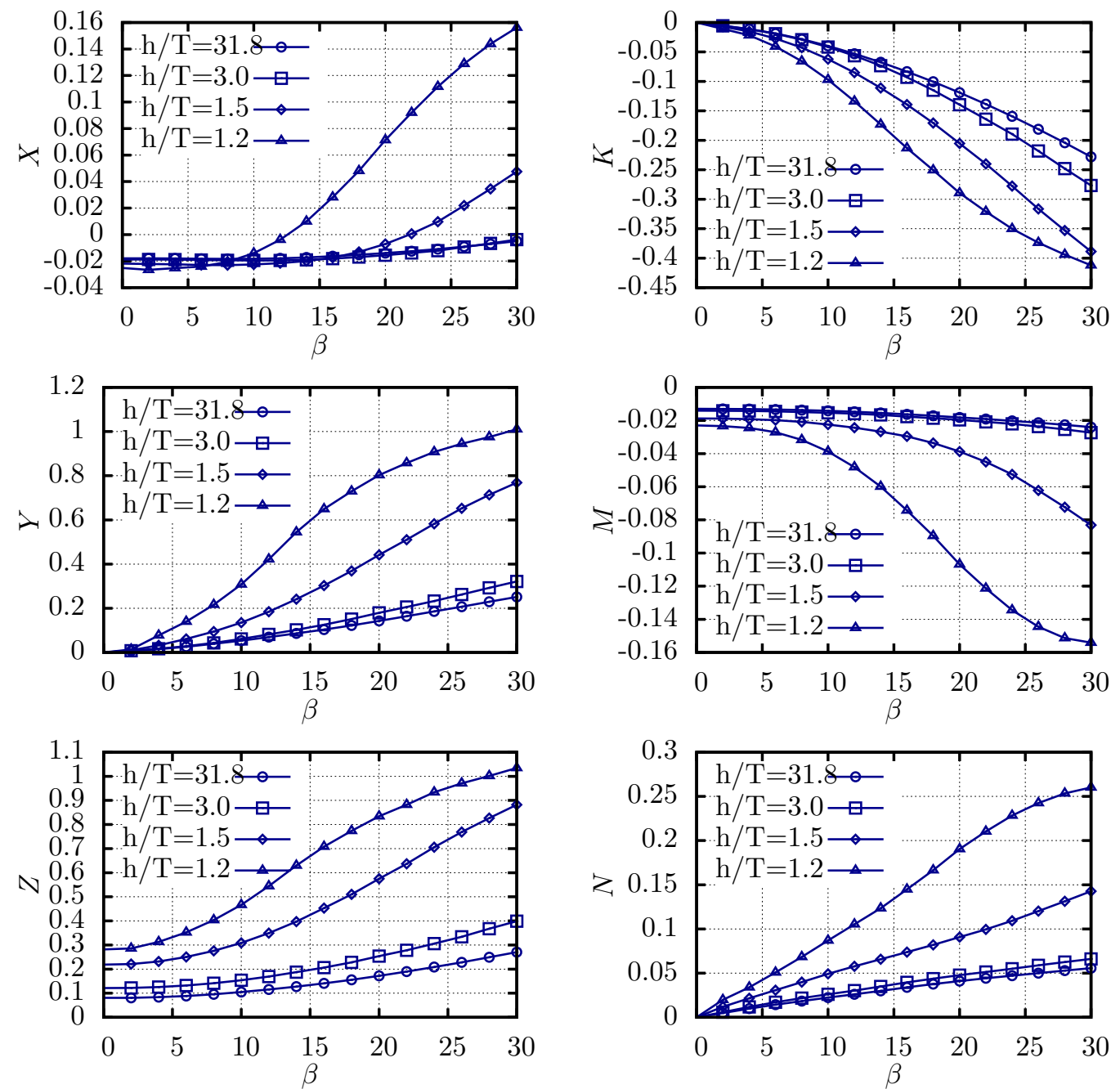

Figure 7: Influence of drift angle and water depth

and the rotation rate is much more complex, however. Noteworthy is the trend observed in the yaw moment $N$ : for yaw rates below $\gamma=0.4$, the influence of the water depth is hardly visible, while a considerable increase in yaw moment is found for the larger yaw rates when the water depth is reduced. Unfortunately, the influence of yaw rate on forces and moments in shallow water has hardly been published by other authors and therefore it cannot be determined whether this is a modelling error or a physical feature. Therefore, more attention to rotational motion in future studies is strongly recommended.

During free sailing tests with the KVLCC2, see Quadvlieg and Brouwer [24], the maximum drift angle and non-dimensional yaw rate during the manoeuvres were respectively $\beta \approx 20^{\circ}$ and $\gamma \approx 0.8$. Figure 7 and Figure 8 indicate that for these conditions the influence of the water depth on the forces and moments is much larger for a fixed drift angle than for a given yaw rate: e.g. the yaw moment for $\beta=20^{\circ}$ increases by a factor of 4.7 between deep and very shallow water, while for $\gamma=0.6$ the increase is only by a factor of 1.6 . 

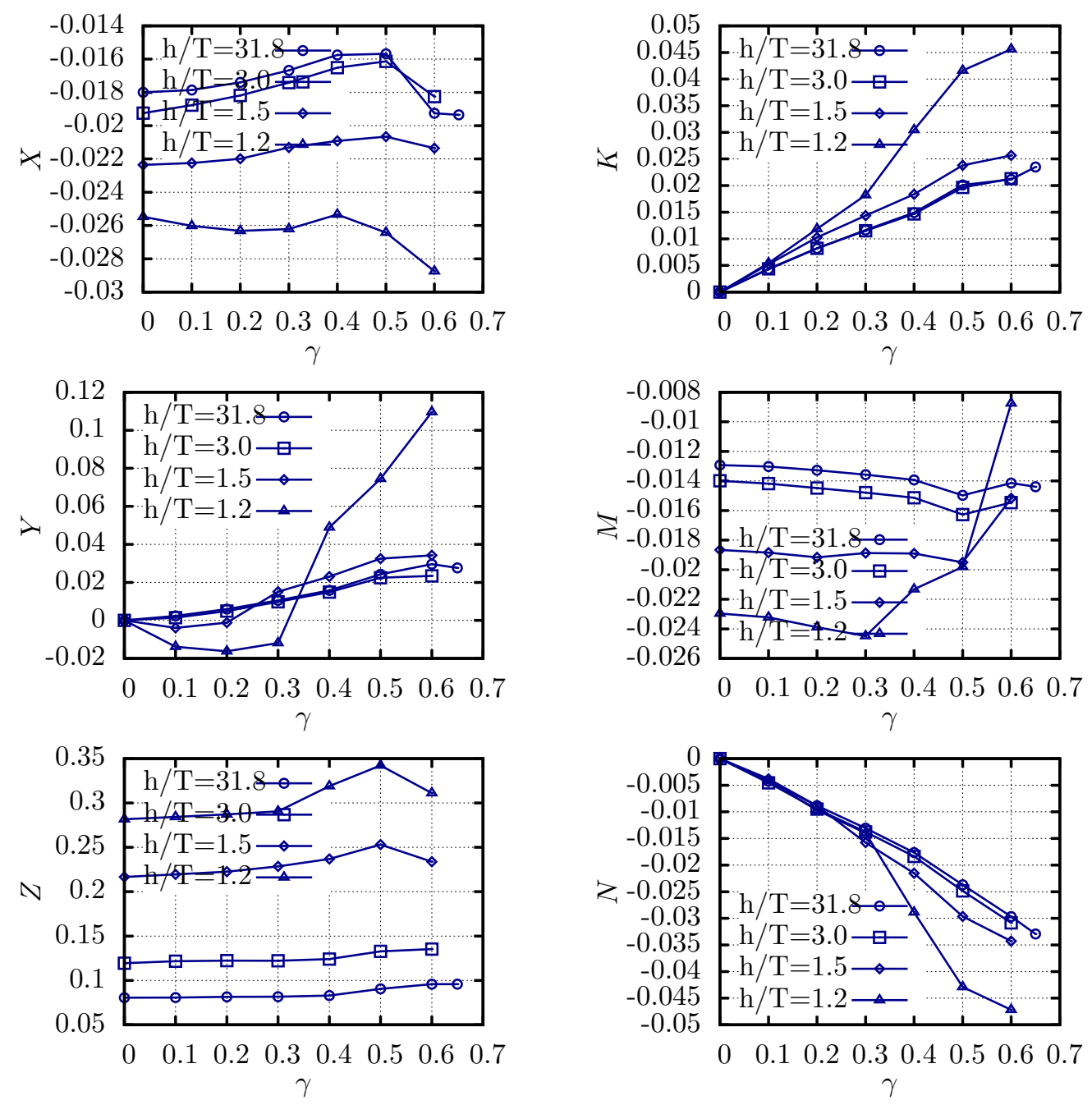

Figure 8: Influence of yaw rate and water depth

\subsubsection{Form factor}

To extrapolate the resistance obtained during model tests to full scale values, use is commonly made of a parameter called the form factor, see e.g. Larsson and Raven [25]. The form factor $(1+k)$ is the ratio between the viscous resistance of the hull and the frictional resistance of a flat plate with the same length and wetted surface area. The form factor is assumed to be the same for model scale as for full scale. In experiments, this factor is determined for each individual hull form from low speed resistance measurements where the wave resistance components are supposed to vanish according to a certain rule:

$$
1+k=\lim _{F n \rightarrow 0} \frac{R}{R_{F}}
$$

The flat plate resistance $R_{F}$ is determined by the formula $R_{F}=\frac{1}{2} \rho V^{2} S_{w a} C_{F}$, with $S_{w a}$ the wetted surface. The coefficient of frictional resistance $C_{F}$ is often determined using 
the ITTC-1957 formula for model-ship correlation:

$$
C_{F}=\frac{0.075}{(10 \log (R e)-2)^{2}}
$$

All calculations in this study have been performed with the double-body assumption which corresponds to the case $F n \rightarrow 0$. Therefore, the obtained resistance can be used directly to calculate the form factor. For a drift angle of $\beta=0^{\circ}$, i.e. straight-ahead sailing, the relation between the water depth and the form factor as presented in Figure 9 is found. Note that the presentation is given using two different parameters on the horizontal axis: the form factor is given as a function of the water depth to draught ratio $h / T$ and as a function of $T /(h-T)$ in the left and right graphs respectively. It is seen that in the latter presentation the points do not all collapse near the $h / T=0$ axis and a clearer relation between the influence of the water depth on the form factor is found.
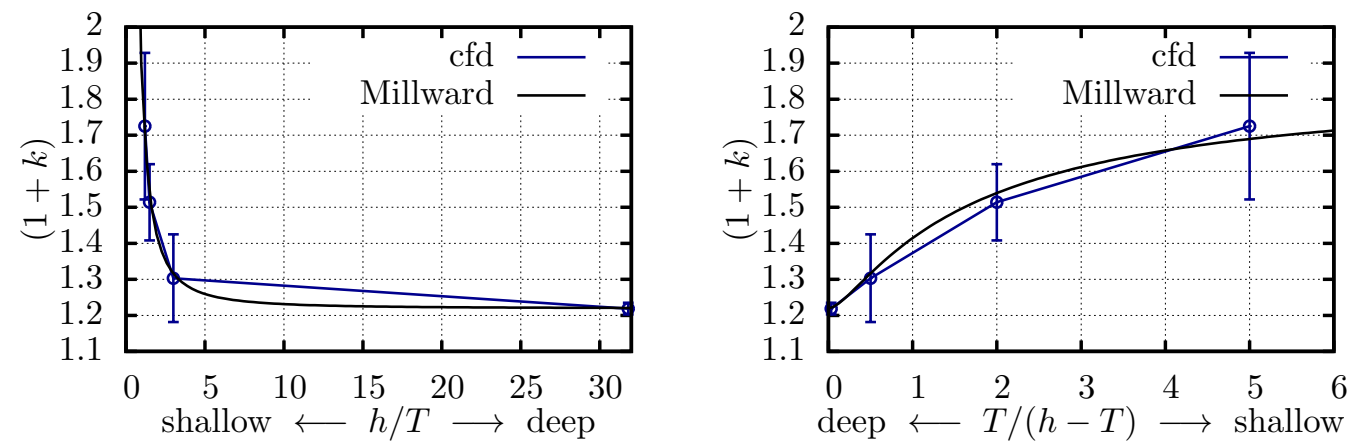

Figure 9: Influence of water depth on form factor

The form factor as calculated with the viscous flow calculations changes from about 1.22 in deep water to 1.73 in very shallow water. Assuming the same form factor on model scale and full scale, this clearly shows that resistance extrapolation to full scale for shallow water conditions cannot be done using a form factor that was obtained in deep water. Millward [26] published a method to correct form factors found for deep water to shallow water conditions. According to Millward, the increase of the form factor due to shallow water is:

$$
\Delta k=0.644\left(\frac{T}{h}\right)^{1.72}
$$

For $h / T=1.2$, this amounts to $\Delta k=0.471$, such that the form factor becomes $(1+k)=$ $1.22+0.471=1.69$ which is reasonably close to the form factor found in the CFD calculation for this water depth. The relation between the form factor based on Millward's formula and the water depth has been plotted in the graph. The agreement between Millward's formula and the form factors predicted by CFD is encouraging. It is therefore recommended to investigate whether the form factor correction proposed by Millward can be applied to other ships as well. Furthermore, studies are currently conducted at MARIN to investigate whether the form factor on model scale is the same as the form factor on full scale. 


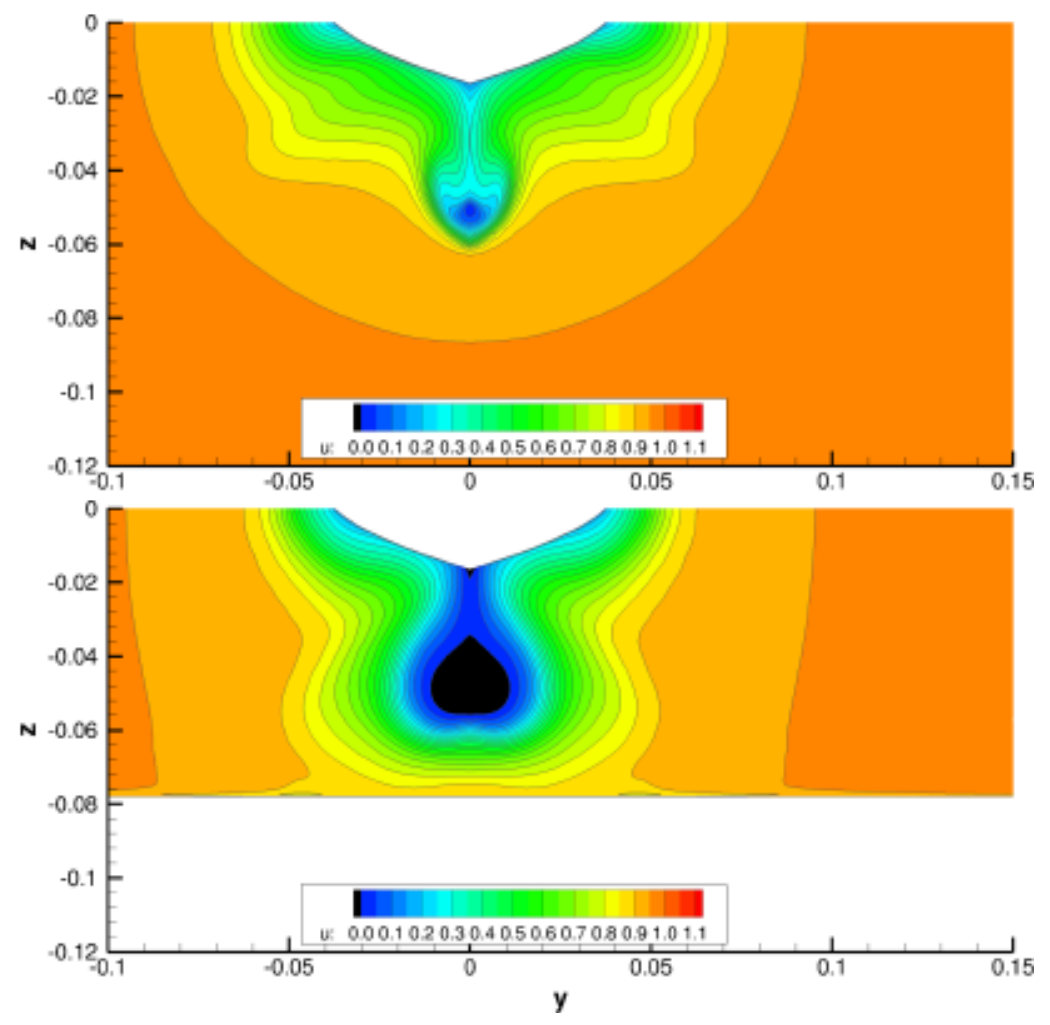

Figure 10: Axial velocity in deep (top) and very shallow (bottom) water, $\beta=0^{\circ}$

\subsection{Local quantities}

In Figures 10 through 12, flow fields around the hull are presented for deep and very shallow water, with $\beta=0^{\circ}, \beta=4^{\circ}$ and $\gamma=0.4$. After examination of the flow fields for these conditions and for the other drift angles and rotation rates, it is observed that for reducing water depth, the pressures below the hull decrease, while the velocities increase (which is according to expectations). Flow separation is detected in restricted water, just below the propeller hub, see Figure 10. The flow separation at the aft ship increases and grows upward for decreasing water depths. For straight ahead condition, the flow separation is much more pronounced than for the drift or rotation condition. The wake near the water surface does not change significantly when changing the water depth. However, the wake around the propeller hub becomes thicker as the water depth reduces. Furthermore, the wake starts to extend to the sea floor, resulting in bulging contour lines. The vortices developing around the aft body, responsible for the famous hook shape in the propeller wake plane, are less concentrated at reduced water depth, which is demonstrated in more detail in Figure 13.

\section{CONCLUSIONS}

An elaborate numerical study has been conducted for the KVLCC2 hull form sailing in various water depths. The calculations give clear insight into the forces and moments acting on the ship as a function of drift angle, yaw rate and water depth. Uncertainty estimates were made for the various calculations. In general, the uncertainties increase 


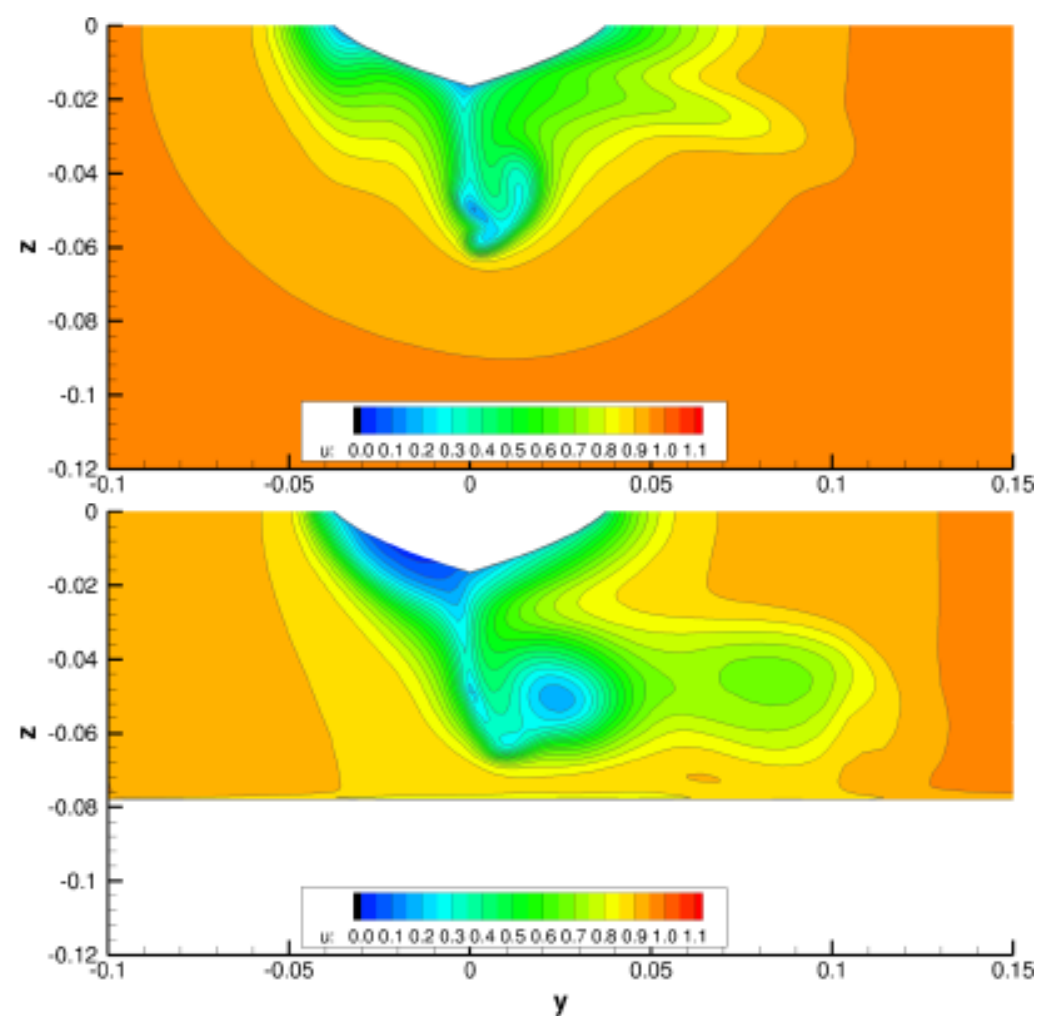

Figure 11: Axial velocity in deep (top) and very shallow (bottom) water, $\beta=4^{\circ}$

with increased flow complexity, i.e. for larger drift angles or yaw rates. A dependency of the uncertainty on the water depth was not found.

The correspondence between the experiments and the calculations is qualitatively reasonable. Quantitatively, it is difficult to draw conclusions because of the scatter in the INSEAN experimental data. Additional measurements, with a larger range of drift angles and rotation rates and combinations thereof, are very much desired. Comparisons between resistance measurements by MOERI and the computations show a good correlation, with a comparison error of the same order of magnitude as the validation uncertainty. The resistance predicted by REFRESCO is therefore judged to be good. By varying the settings of the turbulence modelling, the comparison error could be cancelled completely. This clearly demonstrated the sensitivity of the results to the turbulence modelling.

For steady drift conditions, a clear dependence of the forces and moments on the water depth was demonstrated. For pure rotation, this dependence is much more complex and only develops fully for non-dimensional rotation rates above $\gamma=0.3$. Further study is required to investigate this phenomenon. The influence of the water depth on the relation between the forces and moments and the drift angle is found to be much larger than the relation with the rotation rate.

Based on the results, form factors for deep and shallow water were derived. The calculated form factors change from about 1.22 in deep water to 1.73 in shallow water. This clearly shows that resistance extrapolation to full scale for shallow water conditions cannot be done using a form factor that was obtained in deep water. It was found that for the KVLCC2 the trends closely follow the empirical formula proposed by Millward. 


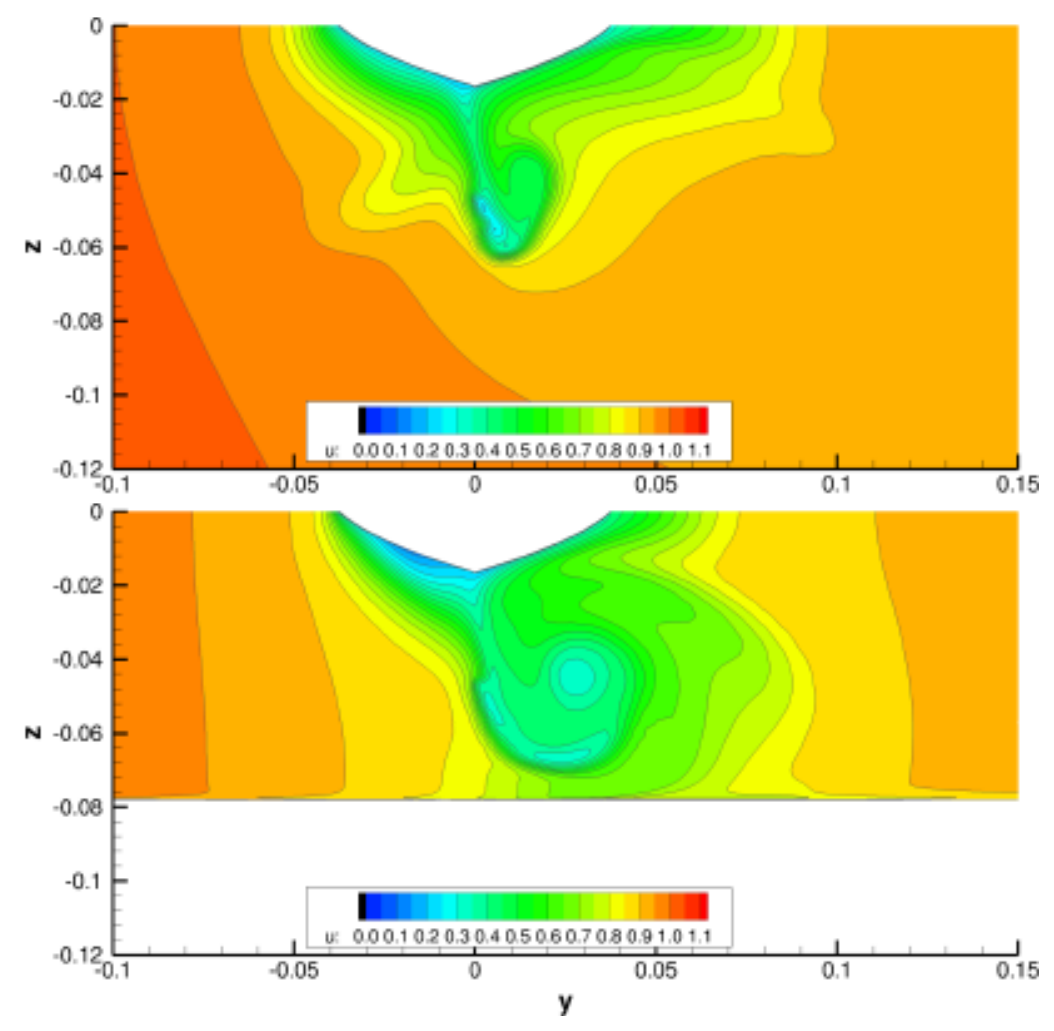

Figure 12: Axial velocity in deep (top) and very shallow (bottom) water, $\gamma=0.4$
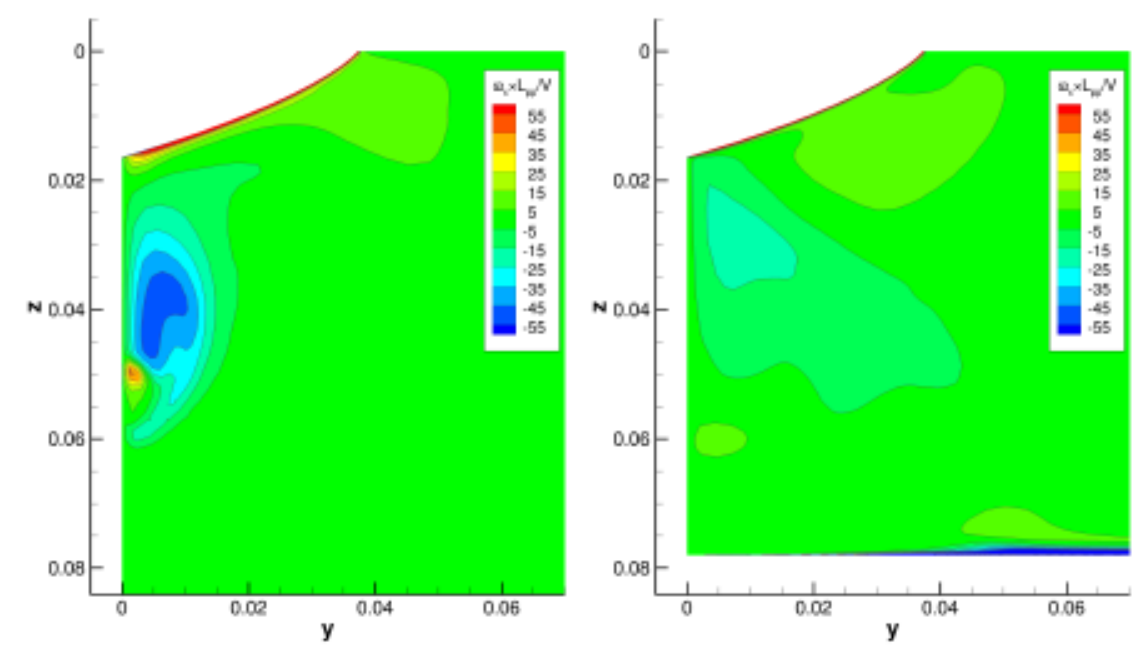

Figure 13: Axial vorticity in deep (left) and very shallow (right) water, $\beta=0^{\circ}$

Future research is recommended to investigate whether the Millward correction can be applied to other hull forms as well.

In shallow water, the flow separation at the stern increases. For straight ahead condition, the flow separation is more pronounced than in manoeuvring conditions. The aft body vortex, responsible for the hook shape in the propeller wake plane, reduces considerably in reducing water depth. 


\section{REFERENCES}

[1] Stern, F. and Agdrup, K., editors. SIMMAN Workshop on Verification and Validation of Ship Manoeuvring Simulation Methods, Copenhagen, Denmark, April 2008.

[2] Toxopeus, S. L., Simonsen, C. D., Guilmineau, E., and Visonneau, M. Viscous-flow calculations for KVLCC2 in manoeuvring motion. In AVT-189 Specialists Meeting on Assessment of Stability and Control Prediction Methods for NATO Air and Sea Vehicles, Portsdown West, UK, October 2011.

[3] Larsson, L., Stern, F., and Bertram, V. Benchmarking of computational fluid dynamics for ship flows: The Gothenburg 2000 workshop. Journal of Ship Research, 47(1):63-81, March 2003.

[4] Larsson, L., Stern, F., and Visonneau, M., editors. Gothenburg 2010: A Workshop on Numerical Ship Hydrodynamics, Gothenburg, Sweden, December 2010.

[5] Lee, S.-J., Kim, H.-R., Kim, W.-J., and Van, S.-H. Wind tunnel tests on flow characteristics of the KRISO 3,600 TEU containership and 300k VLCC double-deck ship models. Journal of Ship Research, 47(1):24-38, March 2003.

[6] Kim, W. J., Van, S. H., and Kim, D. H. Measurement of flows around modern commercial ship models. Experiments in Fluids, 31(5):567-578, November 2001.

[7] Fabbri, L., Benedetti, L., Bouscasse, B., Gala, F. L., and Lugni, C. An experimental study of the maneuverability of a blunt ship: the effect of the water depth. In International Conference on Ship and Shipping Research (NAV), Genova, June 2006.

[8] Fabbri, L., Benedetti, L., Bouscasse, B., Gala, F. L., and Lugni, C. An experimental study of the maneuverability of a blunt ship: the effect of the water depth. In $9^{\text {th }}$ Numerical Towing Tank Symposium (NuTTS), Le Croisic, France, October 2006.

[9] Campana, E. and Fabbri, L. An experimental study of the manoeuverability of a blunt ship: The effect of the water depth. In AVT-189 Specialists Meeting on Assessment of Stability and Control Prediction Methods for NATO Air and Sea Vehicles, Portsdown West, UK, October 2011.

[10] Vaz, G. N. V. B., Jaouen, F. A. P., and Hoekstra, M. Free-surface viscous flow computations. Validation of URANS code FRESCo. In $28^{\text {th }}$ International Conference on Ocean, Offshore and Arctic Engineering (OMAE), number OMAE2009-79398, Honolulu, Hawaii, May 31June 52009.

[11] Vaz, G. N. V. B., Waals, O., Fathi, F., Ottens, H., Le Souef, T., and Kwong, K. Current Affairs - model tests, semi-empirical predictions and CFD computations for current coefficients of semi-submersibles. In $28^{\text {th }}$ International Conference on Ocean, Offshore and Arctic Engineering (OMAE), number OMAE2009-80216, Honolulu, Hawaii, May 31-June 52009.

[12] Vaz, G. N. V. B., Toxopeus, S. L., and Holmes, S. Calculation of manoeuvring forces on submarines using two viscous-flow solvers. In $29^{\text {th }}$ International Conference on Ocean, Offshore and Arctic Engineering (OMAE), number OMAE2010-20373, Shanghai, China, June 2010 . 
[13] Koop, A. H., Klaij, C. M., and Vaz, G. N. V. B. Predicting wind shielding for FPSO tandem offloading using CFD. In $29^{\text {th }}$ International Conference on Ocean, Offshore and Arctic Engineering (OMAE), number OMAE2010-20284, Shanghai, China, June 2010.

[14] Toxopeus, S. L. Practical application of viscous-flow calculations for the simulation of manoeuvring ships. PhD thesis, Delft University of Technology, Faculty Mechanical, Maritime and Materials Engineering, May 2011.

[15] Menter, F. R. Two-equation eddy-viscosity turbulence models for engineering applications. AIAA Journal, Vol. 32(8):1598-1605, August 1994.

[16] Dacles-Mariani, J., Zilliac, G. G., Chow, J. S., and Bradshaw, P. Numerical/experimental study of a wingtip vortex in the near field. AIAA Journal, 33:1561-1568, September 1995.

[17] Bettle, M. C., Toxopeus, S. L., and Gerber, A. G. Calculation of bottom clearance effects on Walrus submarine hydrodynamics. International Shipbuilding Progress, 57(3-4):101-125, 2010 .

[18] Eça, L., Vaz, G. N. V. B., and Hoekstra, M. A verification and validation exercise for the flow over a backward facing step. In Fifth European Conference on Computational Fluid Dynamics, Lisbon, Portugal, June 2010. ECCOMAS.

[19] Eça, L., Hoekstra, M., and Raven, H. C. Calculation of the flow around the KVLCC2 tanker: Case 1.1.a. In Gothenburg 2010: A Workshop on CFD in Ship Hydrodynamics, pages 547-552 (Preprints), Gothenburg, Sweden, December 2010.

[20] Zou, L. CFD predictions including verification and validation of hydrodynamic forces and moments on a ship in restricted waters. Licentiate thesis, Chalmers University of Technology, Gothenburg, Sweden, June 2011.

[21] Simonsen, C. D., Stern, F., and Agdrup, K. CFD with PMM test validation for manoeuvring VLCC2 tanker in deep and shallow water. In International Conference on Marine Simulation and Ship Manoeuvring (MARSIM), number M04, Terschelling, The Netherlands, June 2006.

[22] Eça, L. Calculation of the flow around the KVLCC2 tanker at model and full scale Reynolds numbers with eddy-viscosity models. IST Report D72-43, Lisbon, Portugal, January 2007.

[23] Kume, K., Hasegawa, J., Tsukada, Y., Fujisawa, J., Fukasawa, R., and Hinatsu, M. Measurements of hydrodynamic forces, surface pressure, and wake for obliquely towed tanker model and uncertainty analysis for CFD validation. Journal of Marine Science and Technology, 11(2):65-75, June 2006.

[24] Quadvlieg, F. H. and Brouwer, J. KVLCC2 benchmark data including uncertainty analysis to support manoeuvring predictions. In IV International Conference on Computational Methods in Marine Engineering (MARINE), Lisbon, Portugal, September 2011.

[25] Larsson, L. and Raven, H. C. Ship resistance and flow. In The Principles of Naval Architecture. SNAME, 2010.

[26] Millward, A. The effect of water depth on hull form factor. International Shipbuilding Progress, 36(407):283-302, 1989. 